\title{
Imaging of cardiac amyloidosis: Will this become a unique application for dual-isotope imaging?
}

\author{
Sarah Cuddy, $M D,{ }^{\mathrm{a}, \mathrm{b}, \mathrm{c}}$ Sharmila Dorbala, $M D, M P H,{ }^{\mathrm{a}, \mathrm{b}, \mathrm{c}}$ \\ and Marcelo F. Di Carli, MD ${ }^{\mathrm{a}, \mathrm{b}, \mathrm{c}, \mathrm{d}}$ \\ a Cardiovascular Imaging Program, Departments of Medicine and Radiology, Brigham and \\ Women's Hospital, Harvard Medical School, Boston, MA \\ b Division of Nuclear Medicine and Molecular Imaging, Department of Radiology, Brigham and \\ Women's Hospital, Harvard Medical School, Boston, MA \\ c Division of Cardiovascular Medicine, Department of Medicine, Brigham and Women's \\ Hospital, Harvard Medical School, Boston, MA \\ d Brigham and Women's Hospital, Boston, MA
}

Received Apr 15, 2019; accepted Apr 16, 2019

doi: 10.1007/s12350-019-01754-4

\section{See related article, pp. 28-37}

Cardiac amyloidosis is an infiltrative cardiomyopathy characterized by the deposition of amyloid fibrils within the extracellular space of the heart, which is caused by the misfolding of precursor proteins, most commonly transthyretin (ATTR) - a transport protein synthesized in the liver-or monoclonal light chains (AL), produced by an abnormal plasma cell clone. ${ }^{1}$ Identifying the type of amyloid deposition has very important treatment and prognostic implications. ${ }^{2,3}$ Echocardiography and cardiac magnetic resonance imaging are powerful tools for diagnosing cardiac amyloidosis. ${ }^{4,5}$ However, neither technique can reliably differentiate between ATTR and AL forms of cardiac amyloidosis. Despite initial promise in the late 1970s, bone scintigraphy for the detection of cardiac amyloidosis did not gain a hold in clinical practice. One reason was a perceived lack of sensitivity, as the difference in radiotracer avidity between ATTR and AL amyloidosis was not appreciated. However, the more recent demonstration that bone-avid radiotracers are

Reprint requests: Marcelo F. Di Carli, MD, Brigham and Women s Hospital, ASB-L1 037C, 75 Francis St, Boston, MA, 021155; mdicarli@partners.org

J Nucl Cardiol 2020;27:38-40.

$1071-3581 / \$ 34.00$

Copyright (c) 2019 American Society of Nuclear Cardiology. specifically taken up in hearts of patients with ATTR amyloidosis has renewed clinical interest in this diagnostic approach as it is relatively inexpensive, broadly available, and it has a high sensitivity and specificity in the correct setting. ${ }^{6,7}$ In fact, a recent multicenter study demonstrated that bone scintigraphy has a specificity and positive predictive value for cardiac ATTR amyloidosis of $100 \%$ when there is intense myocardial radiotracer uptake (equal or greater than bone) and absence of a monoclonal protein in serum or urine. ${ }^{8}$ Indeed, bone scintigraphy has now largely replaced the need for endomyocardial biopsy in a majority of patients with suspected cardiac amyloidosis. Furthermore, the recent FDA approval of novel therapies designed to treat systemic ATTR amyloidosis and the early promising results in patients with cardiac involvement assigns a critical role to bone scintigraphy in differentiating $\mathrm{AL}$ from ATTR amyloidosis and guiding patient management. ${ }^{9,10}$

Quantification of the intensity of radiotracer uptake is key in the diagnosis of cardiac ATTR amyloidosis using bone scintigraphy. The intensity of retention of bone-avid radiotracers in the heart can be interpreted by semi-quantitative visual analysis, by grading myocardial uptake to rib uptake on planar or SPECT imaging, and/ or by quantifying radiotracer uptake using a heart-tocontralateral lung (H/CL) ratio. Current diagnostic criteria include visual myocardial uptake equal or greater than that in bone (ribs) or a $\mathrm{H} / \mathrm{CL}$ ratio $>1.6 .^{11,12}$ However, the accuracy of either quantitative approach can be affected by blood pool radioactivity, which can lead to equivocal interpretation especially between low- 
grade myocardial uptake and negative scans on planar or SPECT imaging.

In this issue of the Journal, Tamarappoo and colleagues present a simultaneous dual-isotope approach using 99mTc-PYP and Tl-201 SPECT as a method to improve discrimination between AL and ATTR amyloidosis. They hypothesized that the addition of Tl-201 can help delineate myocardial boundaries from blood pool, thereby decreasing the frequency of equivocal scan interpretations. They retrospectively evaluated 112 consecutive patients undergoing dual-isotope imaging for the evaluation of cardiac amyloidosis on clinical grounds. Dual-isotope imaging has been routine at their center for this application. In the 112 patients, there were 39 with ATTR amyloidosis, 26 with AL amyloidosis, and 47 patients without amyloidosis. The images were interpreted visually and quantitatively using the H/ $\mathrm{CL}$ ratio first using the single-isotope scan (i.e., PYP alone) and then adding the information from the dualisotope scan. By expert visual analysis, the dual-isotope approach resulted in a decrease of low-grade myocardial uptake/equivocal scans in the patients with AL amyloidosis from $34 \%$ to $8 \%$ and in the no amyloidosis group from $16 \%$ to $4 \%$, which were read as definitely negative with the addition of Tl-201. In patients with ATTR amyloidosis, only one patient had an equivocal scan when read as single isotope (PYP alone) that was changed to definitely abnormal with the addition of Tl201. By quantitative analysis of H/CL ratio, there were no statistically significant differences between the single- and dual-isotope approach. The area under the ROC curve (AUC) for detection of ATTR by the dual isotope compared to the single-isotope method improved for both the visual $(0.94$ vs 0.84 , respectively, $P<0.01)$ and quantitative (0.95 vs 0.92 , respectively, $P=0.016$ ) analyses. Of note, there was no significant difference in the AUC between the visual and quantitative analysis using the dual-isotope method $(P=0.34)$.

The innovative use of dual-isotope imaging in cardiac amyloidosis and the rigorous comparison to current standard care are particular strengths of this study. At first glance, the findings of the study suggest that this technique may have a limited role in the diagnosis of ATTR amyloidosis as there was only one patient that was reclassified from equivocal to abnormal using dualisotope imaging. This is likely influenced by the fact that most of the clinical experience with bone scintigraphy in cardiac amyloidosis has been obtained in patients with rather advanced stages of the disease when the amyloid burden in the heart, and hence radiotracer uptake, is rather high. Diagnosis at this disease stage seems straightforward even with the single-isotope approach, as shown in a large multicenter study ${ }^{8}$ and now confirmed in this study. However, it is possible that this may change as we move the use of bone scintigraphy to screen populations at risk without overt signs and symptoms of cardiac amyloid involvement. The expansion of the utilization of bone scintigraphy for detection of cardiac amyloidosis earlier in the natural history of the disease in at-risk populations with presumably lower amyloid burden in the heart may require a revision of diagnostic criteria as low-grade tracer uptake in that setting may reflect early ATTR involvement. This is highlighted by the fact that even late in the natural history of the disease, up to $12 \%$ of patients with biopsy proven ATTR cardiac amyloidosis show low-grade myocardial uptake (grade 1 ) on bone scintigraphy. ${ }^{8}$ Under those circumstances, quantitative methods to reduce uncertainty and improve accuracy as described in this paper may become very important.

The use of hybrid SPECT/CT systems may have an advantage over SPECT-only systems to reduce equivocal scan interpretations as the CT will not only provide a means for attenuation correction but may also help improve definition of the myocardial boundaries. However, most laboratories performing nuclear cardiology testing do not have access to SPECT/CT imaging. Conversely, disadvantages of the dual-isotope approach include the added cost and image post-processing time. Radiation exposure with dual-isotope imaging is also considerably higher than with single-isotope imaging (approximately 15 vs $4 \mathrm{mSv}$ ). The comparative clinicaland cost-effectiveness of hybrid SPECT-CT single-isotope imaging vs dual-isotope SPECT imaging will have to be studied to inform best practice.

Is there a potential role for amyloid-targeted imaging agents? There are several amyloid-specific PET tracers, already approved for brain imaging in Alzheimer's disease that are under investigation in cardiac amyloidosis, including ${ }^{11} \mathrm{C}-\mathrm{Pittsburgh}$ compound-B (PiB), ${ }^{18} \mathrm{~F}$-florbetaben, and ${ }^{18} \mathrm{~F}$-florbetapir. ${ }^{13-15}$ Although preliminary experience with this class of imaging agents demonstrates high sensitivity, especially for early disease detection, they do not allow clear differentiation between AL and ATTR cardiac amyloidosis. So, their routine use would require a dualisotope imaging approach that combines bone scintigraphy and amyloid-targeted PET imaging. While this would provide comprehensive phenotyping of disease, it would be a rather expensive approach and it is unlikely to become a cost-effective screening strategy.

In summary, the study by Tamarappoo and colleagues provides a potentially powerful approach to imaging of cardiac amyloidosis. This proof of concept study is intriguing and hypothesis generating and may have important diagnostic implications with the expected growth in the utilization of bone scintigraphy as a screening tool in at-risk populations, especially because 
the efficacy novel therapies for ATTR would be enhanced by early disease detection. Ultimately, a change in the current practice of imaging for cardiac amyloidosis will require multicenter comparative effectiveness studies in larger populations.

\section{Disclosure}

Dr. Cuddy is the recipient of an investigator-initiated research grant from Pfizer. Dr. Dorbala receives consulting fees: Pfizer, GE Health Care, AAA, and an investigatorinitiated grant from Pfizer. Dr. Di Carli is the recipient of investigator-initiated grants from Spectrum Dynamics and Gilead Sciences, and consulting fees from Sanofi Aventis and GE healthcare.

\section{Funding}

Dr. Dorbala is supported in part by funding from $\mathrm{NIH}$ (HL 130563) and the American Heart Association (AHA 16 CSA 2888 0004). Dr. Di Carli is supported in part by funding from NIH (HL132021).

\section{References}

1. Falk RH. Cardiac amyloidosis. Circulation 2011;124:1079-85.

2. Kumar S, Dispenzieri A, Lacy MQ, Hayman SR, Buadi FK, Colby $\mathrm{C}$, et al. Revised prognostic staging system for light chain amyloidosis incorporating cardiac biomarkers and serum free light chain measurements. J Clin Oncol 2012;30:989-95.

3. Connors LH, Sam F, Skinner M, Salinaro F, Sun F, Ruberg FL, et al. Heart failure resulting from age-related cardiac amyloid disease associated with wild-type transthyretin: A prospective, observational cohort study. Circulation 2016;133:282-90.

4. Pagourelias ED, Mirea O, Duchenne J, Van Cleemput J, Delforge $\mathrm{M}$, Bogaert J, et al. Echo parameters for differential diagnosis in cardiac amyloidosis. Circ Cardiovasc Imaging 2017;10:e005588.
5. Maceira AM, Prasad SK, Hawkins PN, Roughton M, Pennell DJ. Cardiovascular magnetic resonance and prognosis in cardiac amyloidosis. J Cardiovasc Magn Reson 2008;10:54.

6. Bokhari S, Castaño A, Pozniakoff T, Deslisle S, Latif F, Maurer MS. (99m)Tc-pyrophosphate scintigraphy for differentiating lightchain cardiac amyloidosis from the transthyretin-related familial and senile cardiac amyloidoses. Circ Cardiovasc Imaging 2013;6:195-201.

7. Perugini E, Guidalotti PL, Salvi F, Cooke RMT, Pettinato C, Riva $\mathrm{L}$, et al. Noninvasive etiologic diagnosis of cardiac amyloidosis using 99mTc-3,3-diphosphono-1,2-propanodicarboxylic acid scintigraphy. J Am Coll Cardiol 2005;46:1076-84.

8. Gillmore JD, Maurer MS, Falk RH, Merlini G, Damy T, Dispenzieri A, et al. Nonbiopsy diagnosis of cardiac transthyretin amyloidosis. Circulation 2016;133:2404-12.

9. Solomon SD, Adams D, Kristen A, Grogan M, González-Duarte A, Maurer MS, et al. Effects of patisiran, an RNA interference therapeutic, on cardiac parameters in patients with hereditary transthyretin-mediated amyloidosis. Circulation 2019;139:431-43.

10. Maurer MS, Schwartz JH, Gundapaneni B, Elliott PM, Merlini G, Waddington-Cruz M, et al. Tafamidis treatment for patients with transthyretin amyloid cardiomyopathy. N Engl J Med 2018;379:1007-16.

11. ASNC. Technetium-Pyrophosphate Imaging for Transthyretin Cardiac Amyloidosis. https://www.asnc.org/files/PracticeResource s/PracticePoints/ASNCPracticePoint-99mTechnetiumPyrophosph ateImaging2016.pdf.

12. Castano A, Haq M, Narotsky DL, Goldsmith J, Weinberg RL, Morgenstern R, et al. Multicenter study of planar technetium 99m pyrophosphate cardiac imaging. JAMA Cardiol 2016;1:880.

13. Law WP, Wang WYS, Moore PT, Mollee PN, Ng ACT. Cardiac amyloid imaging with 18 F-florbetaben PET: A pilot study. J Nucl Med 2016;57:1733-9.

14. Dorbala S, Vangala D, Semer J, Strader C, Bruyere JR, Di Carli MF, et al. Imaging cardiac amyloidosis: A pilot study using 18Fflorbetapir positron emission tomography. Eur J Nucl Med Mol Imaging 2014;41:1652-62.

15. Lee S-P, Lee ES, Choi H, Im HJ, Lee MH, et al. 11C-Pittsburgh B PET imaging in cardiac amyloidosis. JACC Cardiovasc Imaging 2015;8:50-9.

Publisher's Note Springer Nature remains neutral with regard to jurisdictional claims in published maps and institutional affiliations. 